\section{A Clinician's Guide to Nuclear Oncology: Practical Molecular Imaging and Radionuclide Therapies}

\author{
N.P. Alazraki, M.J. Shumate, and D.A. Kooby
}

Reston, VA: Society of Nuclear Medicine and Molecular Imaging, 2007, 206 pages, \$39

A Clinician's Guide to Nuclear Oncology was written to educate clinicians about the types of nuclear medicine tests and treatments that are available for specific cancer types. The goal as stated in the book is to provide a guide for staff physicians, residents, and advanced practitioners on the indications and applications of nuclear medicine for diagnosis, staging, and therapy of malignancy.

After an opening guide on how to use the book, there is an initial chapter providing a general description of ${ }^{18} \mathrm{~F}-\mathrm{FDG}$ PET and PET/CT molecular imaging, including how ${ }^{18} \mathrm{~F}-\mathrm{FDG}$ is taken up in cells, indications for examinations, patient preparation, how the tests are performed and reported, pitfalls, and a couple of case studies. Most of the remaining chapters are organized by cancer type, with additional chapters on pediatric cancers and the comparative costs of diagnostic procedures. Appendix 1 covers radiation and its effects, imaging devices in nuclear medicine, and molecular imaging. Appendix 2 covers radionuclide pharmaceuticals for therapies.

As stated in the opening guide, each chapter is subdivided into the following sections: epidemiology, advances in nuclear imaging, staging and prognosis, diagnostic nuclear medicine scans and primary clinical indications, and radiopharmaceuticals. A description of the procedure, information on patient preparation, explanations of the resulting report, and an examination of the treatment and overall impact of nuclear medicine imaging on patient management follows. Remaining sections address what is on the horizon for diagnosis or therapy for this cancer. Finally, case presentations are used to illustrate applications of nuclear imaging for some of the more common malignancies. Relevant references are offered at the end of each chapter for the reader who wishes to delve deeper.

Chapter 2 covers lung cancer, including subdivisions for small cell, non-small cell, bronchoalveolar carcinoma, carcinoid, and mesothelioma. In the "on the horizon" section, the authors discuss apoptosis, tumor angiogenesis, and receptor blocking strategies that may someday be in the nuclear arsenal. Chapter 3 contains a great deal of information on breast cancer, including how therapies are chosen using PET/CT and sentinel lymph node biopsies. In chapter 4 , the focus is prostate cancer, including coverage of ${ }^{111}$ In-capromab pendetide (ProstaScint; EUSA Pharma [USA], Inc.) and bone scanning as well as prognosis and staging. Chapter 5 , on colon cancer, includes information about chemotherapy options and side effects. It explains why some patients need to have a colostomy whereas others are spared that procedure. Chapter 6 covers the 2 major classifications of lymphomaHodgkin disease and non-Hodgkin lymphoma-and how these classifications are subdivided into several others. This chapter also covers therapy with ${ }^{131}$ I-tositumomab (Bexxar; GlaxoSmithKline) and ibritumomab tiuxetan (Zevalin; Spectrum Pharmaceutical, Inc.). Chapter 7 discusses the staging and classifications of melanoma. Chapter 8 covers neuroendocrine cancers and discusses selection of scanning techniques including MIBG, ${ }^{111} \mathrm{In}$-pentetreotide (OctreoScan; Covidien), and ${ }^{18}$ F-FDG PET/CT. Esophageal and gastric cancers are discussed in chapter 9, and pancreatic and hepatobiliary malignancies in chapter 10 . Chapter 11 discusses soft-tissue sarcomas and how they are staged differently from other tumors. Chapter 12 discusses the different types of thyroid cancer, with staging, imaging, and treatment options. Chapter 13 covers head and neck cancers and provides excellent diagrams of the anatomy of lymph nodes in the neck. Chapter 14 thoroughly discusses pediatric cancers. Chapters 15 and 16 cover germ cell (testicular) and gynecologic cancers. Chapter 17 discusses renal, ureteral, and bladder cancers. The different types of brain cancers are the topic of chapter 18. Chapter 19 discusses how nuclear imaging is helpful for the patient with cancers of an unknown primary.

I like the way the authors organized this book. The highlights at the beginning of each chapter provide a quick-glance go-to guide on cancer types. The reader needs to be aware that the Medicare reimbursement information is a bit out of date and that some of the statistics are from the early 2000s. Professionals who will find this book beneficial are those looking for education on how nuclear medicine studies assist in the overall care and management of patients with cancer. Quite often, we know what our scan shows but not what that means to the patient we are scanning. For example, you acquire a PET/CT scan of a patient with non-Hodgkin lymphoma, and there is a mediastinal lymph node positive for uptake from the primary lymphoma in the chest but the rest of the scan is clear. What options for treatment does that patient have? This book goes through each cancer type, stage, and treatment option based on the nuclear medicine scan results. It also goes through survival rates for the various stages of cancer. Although this book focuses heavily on PET, it also includes all nuclear medicine imaging and treatment options for cancer. So if you wish to learn more about the pros and cons of all the different nuclear options such as ${ }^{111}$ In-satumomab pendetide (OncoScint; Cytogen Corp.) imaging or ${ }^{90}$ Y-microspheres, this book delivers. As with anything, the more information you have on a subject the better care you are able to give the patient. This book provides you with a great background on many cancers and the benefits of nuclear imaging and treatment to your patient.

\section{Tricia Peters}

Cancer Center of Santa Barbara

300 W. Pueblo St.

Santa Barbara, CA 93105

E-mail: tricia@ccsb.org

Published online Aug. 13, 2012. DOI: $10.2967 /$ jnmt.112.108597 